\title{
elementos de transmisión de vapor a través de materiales porosos
}

\author{
Francisco J. Gómez Rodríguez, Ledo. en Ciencias Físicas \\ División de Instalaciones del I. E. T. c. c.
}

\section{introducción}

Se admite que el movimiento o paso de vapor a través de un medio poroso viene regido por la ley de Fick:

$$
q_{v}=-k \text { grad. } C:
$$

en la cual el signo menos indica que el vapor se mueve en el sentido de las concentraciones decrecientes.

Y si admitimos, como simplificación, que únicamente hay movimiento en la dirección del eje $X$, entonces :

$$
q_{v}=-k \frac{d C}{d x}
$$

Si se supone que se cumple la ecuación de los gases perfectos,

$$
p V=\frac{m}{M} R T \quad " \quad \frac{m}{V}=C=\frac{M}{R T} p
$$

y sustituyendo, teniendo en cuenta que operamos a temperatura constante, toma la forma:

$$
q_{v}=-\frac{k M}{R T} \frac{d p}{d x}=-\mu \frac{d p}{d x}
$$

Integrando la expresión anterior para el caso de una pared homogénea, de espesor $e$ y presiones de vapor en sus paramentos $p_{1}$ y $p_{2} ;\left(p_{2}>p_{1}\right)$, se obtiene:

$$
\int_{0}^{e} q_{v} d x=-\int_{p_{1}}^{p_{2}} \mu d p \quad " q_{v} e=-\int_{p_{1}}^{p_{2}} \mu d p \quad\left\{\begin{array}{l}
0 \leqslant x \leqslant e \\
p_{1} \leqslant p \leqslant p_{2}
\end{array}\right.
$$

Se comprueba experimentalmente que $\mu=f(p)$ y no puede salir, por tanto, fuera de la integral.

Para los cálculos se recurre a utilizar la fórmula:

$$
q_{v} e=\bar{\mu}\left(p_{1}-p_{2}\right)
$$




\section{notaciones}

$a, b=$ Constantes del flúido y del medio.

$c=$ Constante sin dimensiones, que ha de tener el mismo valor para todos los medios porosos.

$C=$ Concentración en volumen de vapor.

$e=$ Espesor del medio poroso.

$E=$ Energía de activación.

$g=$ Cambio de masa, en gramos.

$h_{r_{1}}, h_{r_{2}}=$ Humedades relativas en la fuente y el sumidero.

$k=$ Coeficiente de proporcionalidad.

$m=$ Masa de vapor.

$M=$ Peso molecular.

$p=$ Presión de vapor.

$p_{2}=$ Presión de vapor para $x=0$

$p_{1}=$ Presión de vapor para $x=e$

$p_{s}=$ Presión de saturación de vapor, a la temperatura de ensayo.

$\boldsymbol{P}=$ Permeancia del material.

$R=$ Constante de los gases.

$R_{k}=$ Número de Reynolds para los medios porosos.

$S=$ Area de la muestra atravesada por el vapor, en metros cuadrados.

$t=$ Tiempo, en horas (durante el cambio $g$ ).

$T=$ Temperatura absoluta.

$v$ = Velocidad macroscópica del flúido.

$\mu=$ Permeabilidad.

$\mu=$ Permeabilidad media.

$\mu_{0}=$ Permeabilidad para $T=\infty$.

$\nu=$ Viscosidad absoluta del flúido.

$\rho=$ Densidad del flúido. 
en donde $\bar{\mu}$ queda definida por:

$$
\mu=\frac{1}{p_{2}-p_{1}} \int_{p_{1}}^{p_{2}} \mu d p
$$

Puede adoptarse también como magnitud representativa del transporte de vapor a través del elemento en cuestión su permeancia $P$, dada por la fórmula:

$$
P=\frac{\bar{\mu}}{\rho}
$$

\section{influencia de la temperatura}

La temperatura ejerce asimismo una gran influencia sobre la permeabilidad. Se han efectuado ensayos a distintas temperaturas, obteniéndose diferentes valores de la permeabilidad para el mismo material en iguales condiciones de humedad relativa.

Es posible describir el efecto de la lemperatura para ciertos materiales teniendo en cuenta la energía de activación. Es éste un concepto, desarrollado en la teoría cinética, para explicar el efecto de la temperatura sobre las reacciones químicas.

La fórmula deducida para determinar la relación entre la temperatura y la permeabilidad de un material es:

$$
\mu=\mu_{0} e^{-E / l i T}
$$

Para emplear esta fórmula en la práctica, se recurre al siguiente artificio:

$$
\log \mu=\log \mu_{0}-\frac{E}{R} \cdot \frac{1}{T}
$$

en la cual, si tomamos como abscisa $\frac{1}{T}$ y como ordenada log $\mu$, resulta ser gráficamente una recta de pendiente $\left(-\frac{E}{R}\right)$ y de ordenada en el origen $\left(\log \mu_{0}\right)$.

Bastará, por tanto, tomar dos medidas experimentales a distinta temperatura para poder dibujar la recta y conocer la permeabilidad del material a cualquier temperatura.

La aplicación de esta ecuación se limita solamente a materiales, tales como las gomas, resinas y polímeros, en los cuales las moléculas de vapor se mueven a través de las estructuras moleculares por un proceso conocido como difusión activada. En los demás casos, se ha de tomar únicamente como un indice de aproximación.

\section{mbtodos experimentales}

Existen numerosos ensayos que tienen por objeto determinar la permeabilidad o permeancia de diferentes materiales de construcción en forma de hojas o láminas de unos pocos milímetros de espesor, tales como láminas plásticas, papeles embetunados, etc. 
Se encuentran normalizados en los distintos paises, of reciendo variantes en cuanto a las condiciones de humedad y temperatura, pero todos se fundan en esencia en el mismo principio básico, el cual consiste en sellar la muestra por sus bordes a la boca de un plato o vasija impermeable al vapor, en la cual se ha introducido previamente una cantidad determinada de agua destilada (ensayo por vía húmeda) o en su lugar un producto desecante (ensayo por vía seca). Los platos se colocan en una cámara acondicionada para mantener la humedad relativa y temperatura constantes.

A causa de la diferencia de humedades relativas existente entre el interior y el exterior de la vasija, el vapor de agua comienza a circular a través de la muestra de ensayo.

Los platos se pesan periódicamente en una balanza de precisión, observándose incrementos o pérdidas de peso según el tipo de ensayo.

De estos incrementos de peso, área de la muestra expuesta al vapor, tiempo de duración y humedades relativas dentro y fuera de la vasija, podemos deducir los valores de la permeancia y permeabilidad del material ensayado.

Incluimos a continuación el proceso de cálculo indicado por la norma E 96-63 T ASTM como aclaración a lo anteriormente expuesto:

Se dibuja la curva pesos-tiempos en un gráfico, eligiendo por lo menos 4 puntos situados en línea recta, en la zona central (condiciones estacionarias). La pendiente de la recta será la cantidad de vapor transmitido en la unidad de tiempo. Dividiendo por el área de la muestra expuesta al paso de vapor, se obtiene:

$$
G=\frac{g \cdot 24}{t \cdot S}\left(\mathrm{~g} / \mathrm{m}^{2} \cdot \mathrm{h} \cdot 24\right)
$$

A partir de aquí se calcula la permeancia $P$ :

$$
P=\frac{G}{\Delta p}=\frac{G}{p_{s}\left(h_{r_{1}}-h_{r_{2}}\right)}
$$

Las humedades relativas de la atmósfera interior de la vasija se toman normalmente como $0 \%$ (vía seca) y $100 \%$ (vía húmeda). La humedad relativa exterior es la que se halla fijada en las condiciones de ensayo.

Si el material es homogéneo, puede calcularse la permeabilidad como el producto de la permeancia por el espesor:

$$
\bar{\mu}=P \cdot e
$$

Como ya hemos dicho, la permeancia y permeabilidad son dependientes de las condiciones ambientales, y no se pueden comparar con las halladas a otras humedades relativas y temperaturas ni extender a otras condiciones que las del ensayo.

Existe además algún método aislado para determinar el paso de vapor a través de elementos de espesor considerable.

El sistema que indicamos como ejemplo constituye, en esencia, un ensayo por vía seca gigante.

Las muestras ensayadas fueron trozos de muro, de 28 pies cuadrados y de 4,5 a 12 pulgadas de espesor. 
Se utilizó el siguiente procedimiento:

Fijada la muestra en un marco de acero, para facilitar su manejo, se introduce en un recinto a temperatura y humedad relativa constantes, hasta conseguir la estabilidad en su contenido de humedad.

A continuación se pasa la muestra a las cámaras de ensayo, las cuales consisten en dos habitaciones contiguas impermeables al vapor y provistas de las instalaciones necesarias para controlar la temperatura y humedad relativa en su interior. La muestra se sella convenientemente en un hueco apropiado existente en la pared común a las dos cámaras.

Manteniendo la misma temperatura constante para ambas, se establece una diferencia de humedades relativas entre ellas, gracias a la cual circulará el vapor de agua a través de la muestra desde la cámara húmeda hacia la más seca. El control de humedad se consigue en esta última haciendo pasar el aire por un desecante, en la medida necesaria para mantener constantes las condiciones.

Registrando a intervalos regulares el aumento de peso experimentado por el desecante, se puede obtener la medida del paso de vapor a través del muro para las condiciones del ensayo.

\section{modernas teorias}

Los ensayos efectuados por vía húmeda, proporcionan valores notoriamente más altos que los obtenidos por vía seca.

Del desacuerdo existente entre los resultados experimentales y los predichos por la teoría, surge la necesidad de profundizar en el fenómeno analizando las causas y principios por los cuales se rige.

La ley de Fick permanece válida solamente para temperatura constante y muy bajas presiones de vapor. Es necesario entonces edificar una teoría más general, que sea utilizable bajo cualesquiera condiciones y se ajuste a los resultados dados por la experiencia.

Aparece entonces una serie de trabajos aislados entre sí que analizan más profundamente el fenómeno, modificando y completando algunos la ley de Fick y otros desarrollados independientemente de ella, pero no parecen tener por ahora una corroboración completa y precisa con los ensayos efectuados.

En un interesante trabajo, J. C. Ward desarroila una ecuación para movimiento de flúidos a través de un medio poroso a partir de la dada por M. Muskat.

Según este último, la ecuación de flujo de un flúido, en el seno de un medio poroso, debe ser representada por:

$$
\frac{d p}{d x}=a v+b v^{2}
$$

J. C. Ward, mediante consideraciones dimensionales, llega a una ecuación de la forma:

$$
\frac{d p}{d x}=\frac{\nu}{\mu} v+\frac{c \rho}{\mu^{2}} v^{2}
$$

A bajas velocidades, el $2 .^{\circ}$ término del $2 .^{\circ}$ miembro de esta ecuación se puede despreciar frente al $1 .^{\circ}$, quedando entonces: 


$$
\frac{d p}{d x}=\frac{\nu}{\mu} v
$$

que es la ecuación de Darcy para régimen laminar.

Para altas velocidades quedaria, por el contrario,

para régimen turbulento.

$$
\frac{d p}{d x}=\frac{c \rho}{\mu^{\frac{1}{2}}} v^{2}
$$

El cociente

$$
\frac{c \rho}{\mu^{\frac{1}{2}}} v^{2}: \frac{\nu}{\mu} v=c R_{k}
$$

en la cual $R_{k}$ es el número de Reynolds para los medios porosos.

La transición del régimen laminar al turbulento se efectúa para $R_{k}=1,8$, y el valor de $c$ hallado para todos los medios es $c=0,550 \pm 0,024$.

(El sistema de unidades empleado en el C.G.S.)

De la ecuación del movimiento, despejando la permeabilidad, se puede escribir:

$$
\mu=\left[\frac{0,550 \rho v^{2}+\sqrt{0,0302 \rho^{2} v^{4}+4 \nu v-\frac{d p}{d x}}}{2 \frac{d p}{d x}}\right]^{2}
$$

La anterior ecuación del movimiento se ha comprobado con distintos medios porosos, usándose en todos los casos el agua como flúido y encontrando valores suficientemente aproximados a los experimentales. Indica su autor que esta misma ecuación debería regir también para los gases, y se plantea, para un próximo futuro, la comprobación experimental de dicho supuesto.

\section{bibliografía}

1) A. Alamín y F. Agumbe: Condensaciones en edificación. Monografía n.o 250 del I.E.T.e.c., 1965.

2) S. C. Changi-N. B. Hutcheon: Dependence of water vapor permeability on temperature and humidity. H.P.A.C., marzo 1956, págs. 149-155.

3) Norma E96-63-T ASTM.

4) R. J. ZABLoDIL: Tests reveal moisture migrations rates through variously constructed walls. H.P.A.C., septiembre 1961, págs. 148-151.

5) J. C. Wand: Turbulent Flow in Porous Media. Journal of the hydraulic division, ASCE, vol. 90, septiembre 1964, págs. 1-12. 